\title{
Hypocomplementemic Urticarial Vasculitis Syndrome
}

\author{
An Interdisciplinary Challenge
}

Wolfgang Grotz, Hideo A. Baba, Jan U. Becker, Martin W. Baumgärtel

\section{SUMMARY}

Background: Chronic urticaria often points the way to the diagnosis of a systemic disease, particularly when urticarial vasculitis can be demonstrated. Hypocomplementemic urticarial vasculitis syndrome (HUVS) is considered to be an independent immunological disease.

\section{Method: Selective literature review and consideration of} the author's own clinical experience.

Results and conclusions: The main manifestation of HUVS is chronic urticarial vasculitis with complement deficiency and the demonstration of $\mathrm{C} 1 \mathrm{q}$ antibody in the serum. Multiple other organs are involved, sometimes severely. The diagnosis is confirmed by skin biopsy, which reveals leukocytoclastic vasculitis as a pathogenetic correlate of this systemic disease. Although HUVS is relatively rare, the medical specialists that might encounter it-ophthalmologists, rheumatologists, nephrologists, dermatologists, general practitioners, and pediatricians-should include it in their differential diagnoses whenever appropriate. Awareness of HUVS and rational diagnostic evaluation will lessen the chance of it being misdiagnosed as another type of systemic immunological disease and will reduce superfluous diagnostic testing in patients suffering from it.

Key words: urticaria, vasculitis, autoimmune disease, hypocomplementemia, chronic disease

Gite this as: Dtsch Arztebl Int 2009; 106(46): 756-63 DOH: 10.3238/arztebl.2009.0756

Klinik für Innere Medizin II/Nephrologie, Alfried Krupp Krankenhaus, Essen: Prof. Dr. med. Grotz

Institut für Pathologie und Neuropathologie, Universitätsklinikum Essen: Prof. Dr. med. Baba

Institut für Pathologie, Medizinische Hochschule Hannover: Dr. med Becker

1. Medizinische Klinik, St. Franziskus Hospital, Münster: Dr. med. Baumgärtel rticaria is a cutaneous vascular reaction with locally increased vascular permeability. It is clinically manifested by erythema and wheals and is accompanied by itching or a burning sensation. The wheals are usually transient, persisting for anything from a few minutes to a few hours. Urticarial vasculitis (UV) is a discrete disease entity, distinct from urticaria, in which the urticarial lesions persist for more than 24 hours and heal leaving brownish residues (1). On diascopy the cutaneous efflorescences generally display a central red spot, betraying the underlying vasculitis. Because leukocytoclastic vasculitis involves leakage of erythrocytes from blood vessels, hyperpigmentation remains after resolution of the urticarial lesion. In contrast, the exanthema seen in acute or chronic urticaria resolves completely within 24 hours (2).

\section{Incidence}

Some $2 \%$ to $20 \%$ of patients admitted to the hospital for investigation of chronic urticaria are found to have UV. Hypocomplementemic urticarial vasculitis syndrome (HUVS), however, a severe systemic form of urticarial vasculitis, is rare; its exact incidence is unknown. In one retrospective study at a single center, $18 \%$ of patients with biopsy-confirmed UV exhibited a hypocomplementemic form of the disease (3). HUVS affects women more frequently than men (ratio 2:1). The peak incidence is seen in the fifth decade of life, but HUVS has also been observed in children (4).

\section{Case report}

A 72-year-old woman had a 6-month history of night sweats, weight loss, worsening general health, urticarial skin lesions, and recurring conjunctivitis. Exhaustive dermatological, gastroenterological, cardiological, hemato-oncological, angiological, and radiological investigations failed to pinpoint the diagnosis. Attempts at treatment with antihistamines, triamcinolone lotions, and prednisolone (10 mg p.o.) were unsuccessful. Severe abdominal cramps and nausea led to hospital admission. 
Physical examination revealed urticarial exanthema with areas of brownish hyperpigmentation on the trunk and extremities (Figure 1) accompanied by bilateral conjunctivitis. The patient was slightly sensitive to left lower abdominal and epigastric pressure and exhibited arthritis in the right ankle joint and first metacarpophalangeal joint that resolved spontaneously after 24 hours. Laboratory tests showed increased erythrocyte sedimentation rate (ESR) and c-reactive protein (CRP), anemia, an elevation of antinuclear antibodies to 1:1280 — without demonstration of double-strand DNA antibodies, anti-extractable nuclear antigen (ENA), or antiphospholipid antibodies_-but weakly positive mixed cryoglobulins with no monoclonal components, strongly decreased complement activity, and raised tumor marker CA125. Examination of the urinary sediment revealed isolated glomerular hematuria.

Gastroscopy, colonoscopy, gynecological examination, abdominal CT and echocardiography showed distinct ascites (granulocyte-rich exudate) and a discrete pericardial effusion with no indication of malignancy. The conjunctivitis was not improved by gentamicin eye drops. Histological examination of a repeat dermal biopsy sample from a fresh urticarial efflorescence on the thigh showed leukocytoclastic vasculitis with immunohistochemical demonstration of C1q deposits on the vascular endothelium. The material used for immune complex diagnosis was fixed in formalin and embedded in paraffin, with primary antibodies to IgA, IgG, IgM, C3c, and C1q. Antigen unmasking with proteases and a polymer-based detection system with horseradish peroxidase were employed to mark the binding sites of the primary antibodies brown. This procedure matches immunofluorescence for sensitivity and specificity. There were no bandlike deposits on the basal membrane. The chronic urticarial efflorescences with histological demonstration of small-vessel vasculitis and the strongly decreased complement factors pointed to the rare clinical picture of HUVS, particularly since all six minor criteria were fulfilled (Box 1).

Monotherapy with $2 \mathrm{~g}$ mycophenolate mofetil in short-term combination with $50 \mathrm{mg}$ prednisolone swiftly resulted in complete remission for more than 24 months. Interestingly, the treatment for vasculitis also normalized the tumor marker level.

UV represents a disease continuum whose lifethreatening end is occupied by HUVS. The UV spectrum embraces the following conditions:

- The mild form of disease with distinct urticarial exanthema and slight or absent systemic manifestations without hypocomplementemia (Figure 1a)

- Various special forms: AHA syndrome (21), Schnitzler syndrome (20), Cogan syndrome (19), Muckle-Wells syndrome (18) (Box 2)

- Life-threatening systemic vasculitis with hypocomplementemia and only mild urticarial skin lesions: HUVS (6).

HUVS is also known as McDuffie syndrome, after the researcher who published the first description (7).

\section{Criteria for diagnosis of HUVS*}

- Major criteria

- Chronic urticarial exanthema

- Hypocomplementemia

\section{- Minor criteria}

- Leukocytoclastic vasculitis

- Arthralgia and arthritis

- Uveitis or episcleritis (or conjunctivitis)

- Glomerulonephritis

- Abdominal pain

- Positive C1q antibody

* modified from (5); HUVS, hypocomplementemic urticarial vasculitis syn-

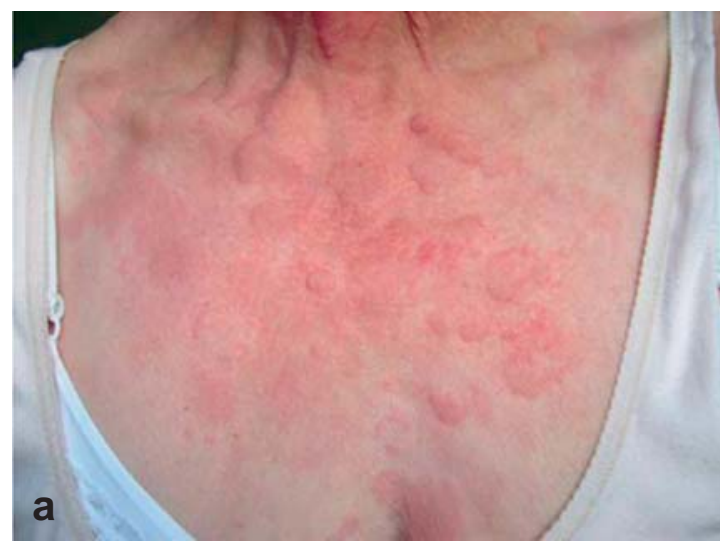

Figure 1

a) Urticarial efflorescences on the upper body in hypocomplementemic urticarial vasculitis syndrome (HUVS)

b) Older efflorescences on the legs of the same patient: reddish hyperpigmentation as expression of urticarial vasculitis 
BOX 2

\section{Special forms of urticarial vasculitis*}

- AHA syndrome

- Arthritis

- Wheals

- Angioedema

- Schnitzler syndrome

- Hyperostosis

- Lymphadenopathy

- Intermittent fever

- Monoclonal IgM gammopathy

- Cogan syndrome

- Interstitial keratitis

- Hypacusis

- Muckle-Wells syndrome

- Deafness

- Renal amyloidosis

${ }^{*}$ modified from (e1); AHA = arthritis, hives, and angioedema

\section{TABLE 1}

Differentiation between urticaria and urticarial vasculitis*

\begin{tabular}{l|l|l} 
& Urticaria & Urticarial vasculitis \\
\hline Pain & - & + \\
\hline Itching & + & + \\
\hline Duration & $<3$ hours & $>24$ hours \\
\hline Resolution & Complete & Hyperpigmentation \\
\hline Diascopy & - & Central red spot \\
\hline Angioedema & - & Possible
\end{tabular}

\section{TABLE 2}

Organ involvement in HUVS*

\begin{tabular}{|c|c|c|}
\hline Skin & $100 \%$ & $\begin{array}{l}\text { Urticarial exanthema, urticarial vasculitis, } \\
\text { palpable purpura, angioedema }\end{array}$ \\
\hline Joints & $70 \%$ & Arthralgia and arthritis \\
\hline Kidneys & $50 \%$ & $\begin{array}{l}\text { Proteinuria, hematuria, renal insufficiency, } \\
\text { rapid progressive glomerulonephritis (RPGN) }\end{array}$ \\
\hline Gastrointestinal tract & $30 \%$ & $\begin{array}{l}\text { Abdominal pain, nausea, vomiting, diarrhea, } \\
\text { hepatomegaly, splenomegaly, ascites, serositis }\end{array}$ \\
\hline Lungs & $20 \%$ & $\begin{array}{l}\text { Shortness of breath, coughing, hemoptysis, } \\
\text { pleural effusion, chronic obstructive pulmonary } \\
\text { disease (COPD) }\end{array}$ \\
\hline Eyes & $10 \%$ & Episcleritis, uveitis, conjunctivitis \\
\hline Heart & Rare & Pericarditis, cardiac valve disease \\
\hline Nervous system & Rare & $\begin{array}{l}\text { Pseudotumor cerebri, aseptic meningitis, } \\
\text { central or peripheral nerve palsy }\end{array}$ \\
\hline
\end{tabular}

* modified from (23); HUVS, hypocomplementemic urticarial vasculitis syndrome
Some authors prefer not to term it a syndrome, referring instead to UV. However, the term HUVS has been widely adopted to distinguish the systemic disease from the purely cutaneous manifestation of UV, and we follow this practice.

\section{Organ involvement}

Hypocomplementemic urticarial vasculitis syndrome is a specific autoimmune disease with involvement of the skin, joints, kidneys, and gastrointestinal tract manifested by vasculitis and polyserositis. Occasionally other organ systems are involved.

\section{Skin}

In contrast to other forms of urticaria, the cutaneous efflorescences in UV are caused by leukocytoclastic vasculitis. The urticarial lesions differ from ordinary urticaria in that the wheals persist for more than 24 hours and leave brown residues after resolution (Table 1).

If the vasculitis also involves deeper vessels, angioedema may be found. Immunohistochemistry demonstrates immunoglobulin and complement deposits in the vessel walls or on the endothelium. Such deposits also occur together with vasculitis in systemic lupus erythematosus, but in lupus patients they are typically found along the basal membrane (the so-called lupus band) (4).

\section{Joints}

Arthralgia and arthritis of various joints are the most frequent systemic manifestations of HUVS. The joint pain wanders and is often no more than transient. The elbows, wrists, knees, and ankles are involved. Joint deformities are possible, in which case the cardiac valves are also affected (8).

\section{Kidneys}

Renal involvement is often only mild, but dialysis may be required (9). The frequently found proteinuria and hematuria with demonstration of acanthocytes (nephritic sediment) is seen histologically as membranous, membranoproliferative, or intra- and extracapillary glomerulonephritis. Nevertheless, renal biopsy is necessary only in proteinuria ( $>1 \mathrm{~g}$ /day) or in acute or chronic progressive renal failure. The renal involvement seems to be more severe in children $(10,11)$.

\section{Lungs}

Lung involvement goes along with shortness of breath, coughing, hemoptysis, pleural effusion, and chronic obstructive pulmonary disease (COPD) and is the most frequent cause of death among HUVS patients. The lungs are affected more severely in smokers (3).

\section{Gastrointestinal tract}

Around $30 \%$ of HUVS patients suffer gastrointestinal symptoms: pain, nausea, vomiting, diarrhea, sometimes also ascites in connection with serositis, hepatomegaly, and splenomegaly (12). 


\section{Other organs}

Ocular involvement may be observed, with uveitis, episcleritis, and also conjunctivitis. The heart and the nervous system are occasionally affected (Table 2).

\section{Pathophysiology}

Although the pathophysiology of HUVS has not been fully clarified, the following cascade seems feasible: IgG antibodies to the collagen-similar regions of $\mathrm{C} 1 \mathrm{q}$ form immune complexes and initiate the cascade. These C1q antibodies and immune complexes are also regularly detected in the plasma of patients with HUVS (13). The immune complexes activate the classical complement pathway into and around the blood vessels. The complement products generally lead to mast cell degranulation. The substances released thereby result in increased vascular permeability with urticaria and/or angioedema (14) and lead to histological demonstration of leukocytoclastic vasculitis in terms of a Coombs and Gell type III reaction.

Interestingly, anti-C1q autoantibodies have also been detected in pulmonary diseases. These antibodies can cross-react with surfactant and may thus be responsible for the lung involvement in HUVS $(15,16)$.

\section{Diagnosis}

Alongside chronic urticarial exanthema, the cornerstone of UV diagnosis is demonstration of leukocytoclastic vasculitis in dermal biopsy samples (17). This involves a leukocytoclastic reaction, vessel wall destruction, and deposits of fibrinogen. Immune complexes and complement should be visible in the blood vessels on immunohistochemical analysis. The more severe the findings, the more pronounced is the perivascular neutrophilic infiltrate (Figure 2).

For the diagnosis of HUVS, the typical cutaneous efflorescences must be accompanied by decreased complement levels. All HUVS patients exhibit extremely low levels of C1q. Through activation of the classical complement pathway, complement factors C3 and C4 are also nearly always markedly reduced. A C1q antibody can regularly be detected but is not specific for HUVS (13) (Box 3). The severity of the disease can be judged not just from the clinical signs and symptoms, but also from the degree of complement reduction.

Depending on what organs are affected, investigations such as abdominal sonography, echocardiography, chest x-ray, and lung function tests are required.

To detect any renal involvement, the urine should be examined by microscopy, protein quantification, and if necessary, protein analysis. If a nephritic syndrome is found, renal biopsy should be considered.

\section{Definition}

The criteria for diagnosis of HUVS were established by Schwartz (5) in 1982 (Box 1).

Angioedema, obstructive lung disease, or various neurological findings may also be present but are not listed among the minor criteria.
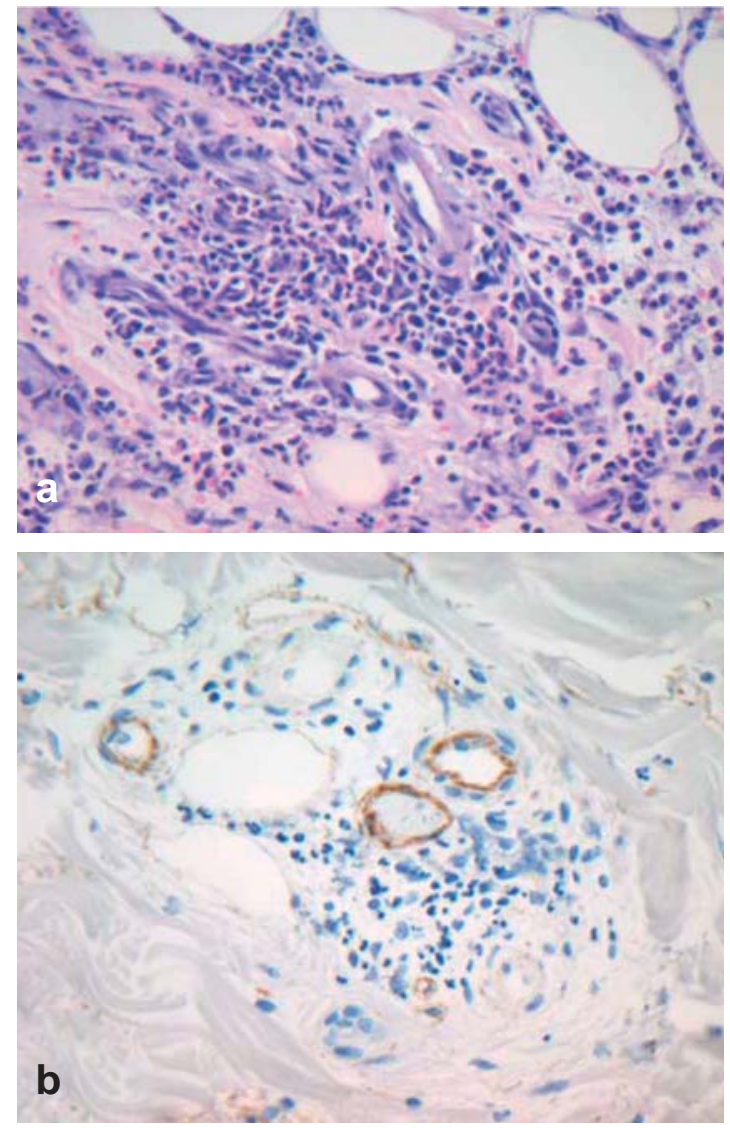

Figure 2

Dermal histology and immunohistology in hypocomplementemic urticarial vasculitis syndrome (HUVS)

a) Dermal perivascular mixed-cell inflammatory cell infiltrate, predominantly granulocytes, with destruction of small vessels and nuclear dust; original magnification $\times 400$

b) Immunohistochemical staining for C1q: demonstration of C1q deposits on the vascular endothelium

\section{BOX 3}

Typical laboratory findings in HUVS*

- ESR acceleration

- Hypocomplementemia with low C1q, C3, C4

- C1q antibodies

- ANA without anti-double-stranded DNA

* modified from (4); HUVS, hypocomplementemic urticarial vasculitis syndrome; ESR, erythrocyte sedimentation rate; ANA, antinuclear antibodies 
Diagnostic criteria for systemic lupus eryhematosus (SLE) by the American College of Rheumatology (ACR)*

\section{- Butterfly erythema}

Fixed erythema, flat or elevated, in the buccal region, usually not involving the nasolabial folds

\section{- Discoid skin changes}

Erythematous, raised skin lesions with adherent keratotic elements and follicular occlusion; atrophic scars can occur in old lesions

\section{- Photosensitivity}

Skin reddening in the course of an unusual reaction to sunlight; occurrence reported by patients

\section{- Oral ulceration}

Oral or nasal ulceration, usually painless; noted by a doctor

\section{- Arthritis}

Non-erosive arthritis involving two or more peripheral joints, characterized by stiffness, swelling, and joint effusion

\section{- Serositis}

a) Pleuritis-typical anamnesis for pleuralgia or pleural fremitus, detected by auscultation by a doctor, or demonstration of a pleural effusion, or

b) Pericarditis—confirmed by ECG or by friction sounds, or by demonstration of a pericardial effusion

\section{- Renal disease}

a) Persisting proteinuria of more than $0.5 \mathrm{~g} /$ day or greater than $3+$, if no quantification is performed, or

b) Cellular cylinders, erythrocyte, hemoglobin, granular, tubular, or mixed cylinders

\section{- Neural disease}

a) Cramps-exclusion of drug induction or a metabolic disturbance, e.g., uremia, ketoacidosis, or electrolyte disturbances, or

b) Psychosis-without obvious drug induction, exclusion of a metabolic disturbance, e.g., uremia, ketoacidosis, or electrolyte disturbances

\section{- Hematological disease}

a) Hemolytic anemia with reticulocytosis, or

b) Leukopenia, less than 4000 leukocytes $/ \mu \mathrm{L}$, demonstrated on two or more occasions, or

c) Lymphopenia, less than $1500 / \mu \mathrm{L}$ on two or more occasions, or

d) Thrombocytopenia, less than $100000 / \mu \mathrm{L}$ without intake of potentially causative medication

\section{- Immunological disease}

a) Anti-DNA: elevated titer of antibodies to native ds-DNA, or

b) Anti-Sm: detection of antibodies to Sm antigens, or

c) Demonstration of antiphospholipid antibodies:

- elevated IgM or IgG anticardiolipin antibodies, or

- demonstration of lupus anticoagulant by the standard test, or

- false-positive serological test for syphilis, positive for more than 6 months, confirmed by means of a Treponema pallidum immobilization test or a fluorescence Treponema antibody absorption test

\section{- Antinuclear antibodies}

Demonstration of an elevated antinuclear antibody titer on immunofluorescence or an equivalent test at a particular time, unconnected with any medication that may be associated with a so-called drug-induced lupus syndrome 
HUVS can be excluded in the presence of persistently distinctly elevated cryoglobulins and corresponding symptoms of primary or secondary cryoglobulinemia, a high-titer double-stranded DNA antibody, decreased C1 esterase inhibitor levels, or a congenital complement defect.

\section{Differential diagnosis}

In any case of urticaria, the first step is to determine whether it is acute or chronic. Chronic urticaria is diagnosed whenever the disease course (either chronic recurring or chronic continuous) goes back 6 weeks or longer. If chronic urticaria is identified, it must then be investigated by means of an autologous serum test, pseudoallergen-poor diet with oral provocation testing, a RAST test, or a search for infection. To identify cases of autoreactive, infection-associated, paraneoplastic, drug intolerance- or drug allergy-related chronic urticaria, patients must undergo tumor screening and temporarily discontinue any regular medication (1).

As a special form of chronic urticaria, diagnosis of UV requires dermal biopsy with demonstration of leukocytoclastic vasculitis and a perivascular neutrophilic infiltrate. If UV is present, one must establish whether it is isolated or exists in the context of a syndrome.

In Muckle-Wells syndrome there is a mutation of the $N L R P 3$ gene on chromosome $1 \mathrm{q} 44$, which codes the protein cryopyrin. Under physiological conditions this protein is activated in the presence of danger and leads to release of interleukin-1. Possibly the mutated protein cannot be inactivated quickly enough, resulting in excessive reactions.

Clinically, besides the UV, once every few weeks the patients display a 12- to 36-hour-long phase of intermittent fever with painful joints, progressive hearing loss, and secondary amyloid A protein amyloidosis with renal involvement (18).

Cogan syndrome preferentially affects young adults in the third decade of life. Following a rheovirus-III infection, antibodies to the virus core protein can cross-react with the inner ear and eye. The clinical presentation resembles that of Menière disease, with fainting attacks, hearing loss, and interstitial keratitis with reddened, painful eyes and visual impairment (19).

In Schnitzler syndrome UV is combined with a monoclonal IgM gammopathy. Apart from intermittent attacks of fever, the clinical manifestation are bone and joint pains and hyperostoses. A lymphoproliferative disease cannot be detected. The pathogenesis and the part played by IgM are unknown (20).

In arthritis, hives, and angioedema (AHA) syndrome, UV is accompanied by angioedema (21). The C1 esterase inhibitor displays no abnormality of concentration or function. Little is known about the causes of AHA syndrome.

If the above-mentioned syndromes have been excluded and hypocomplementemia is present, then the UV has taken a severe course, with multiple organ involvement, and the differential diagnoses are systemic lupus erythematosus (SLE) and the mixed cryoglobulinemias (22). The active forms of all three diseases feature low complement levels and involvement of multiple organs.

Differentiation from SLE is sometimes difficult, because $50 \%$ of patients with HUVS show positive antinuclear antibodies and often also fulfill the classification criteria of the American College of Rheumatology (Box 4).

The patient whose case is presented above, however, fulfilled only three of the 11 SLE criteria. Inversely, in $50 \%$ of patients with confirmed UV the cause is SLE (3). The C1q antibodies are also not specific for HUVS and may be found in one third of patients with SLE (13).

In addition to the ACR criteria, HUVS and SLE can often be differentiated on the basis of double-stranded DNA antibodies and the typical clinical presentations. High titers of double-stranded DNA antibodies or the demonstration of SLE-typical ENA speak against HUVS.

HUVS can be distinguished from Sharp syndrome, another possible differential diagnosis amongst the collagenoses, by demonstration of the U1-RNP antibody.

The differentiation between HUVS and mixed cryoglobulinemia type III also exhibits overlaps, in that cryoglobulins may occur as an epiphenomenon in both SLE and HUVS (22). Differentiation from mixed cryoglobulinemia type II, however, is possible by the demonstration of a monoclonal immunoglobulin in the cryoprecipitate and on the basis of the usually higher cryoglobulin concentration.

\section{Treatment}

The appropriate treatment of UV and HUVS is determined by the severity of the disease. Because the disease spectrum reaches all the way from simple UV with skin lesions without hypocomplementemia to HUVS with severe systemic involvement, the treatment options are similarly diverse. The rarity of the disease means there is little evidence, and the treatment recommendations are based exclusively on reports of single cases or small series.

Antihistamines represent the cornerstone of treatment for patients who have UV with only cutaneous lesions, serving to control the itching. Antihistamines seldom suffice alone, however, because they do not bring the immune complex formation under control, intervene very late in the inflammation cascade, and cannot alter the course of the disease (23).

Glucocorticoids (GC) are the agents most frequently employed to combat the inflammation and immune complex formation. The dosage depends on the severity of the disease. Patients with hypocomplementemia initially almost always require GC. Because of the well-known adverse effects numerous other immunosuppressives are combined with GC, including methotrexate, azathioprine, cyclophosphamide, cyclosporin, and mycophenolate mofetil. In agreement with the observations of investigators from Berlin (24), this 
last-named drug was notably successful in the patient whose case is presented above.

In highly active disease plasmapheresis can also be employed. This reliably reduces the circulating immune complexes, but the effect is only transitory and the symptoms re-emerge a few days later unless $\mathrm{C} 1 \mathrm{q}$ antibody production is blocked by means of immunosuppressives.

Dapsone has also been used in the treatment of HUVS. Because of the wide range of disease severity, however, the role of dapsone has not been clearly delineated. It may be possible to employ dapsone together with GC and an immunosuppressive in a three-pronged approach (25). Newly developed treatment methods have been successfully employed in the special forms of UV. For instance, there have been repeated reports of the successful use of the interleukin-1-receptor antagonist anakinra in Schnitzler syndrome (20) and Muckle-Wells syndrome (18). In contrast, the B-cell antibody rituximab yielded no therapeutic benefit (20).

\section{Conflict of interest statement \\ The authors declare that no conflict of interest exists according to the guidelines of the International Committee of Medical Journal Editors.}

Manuscript received on 28 January 2008, revised version accepted on 21 April 2009

Translated from the original German by David Roseveare.

\section{REFERENCES}

1. Maurer M, Grabbe J: Urticaria: Its History-Based Diagnosis and Etiologically Oriented Treatment [Urtikaria - gezielte Anamnese und ursachenorientierte Therapi]e. Dtsch Arztebl Int 2008; 105(25): 458-65.

2. Guha B, Youngberg G, Krishnaswamy G: Urticaria and urticarial vasculitis. Comp Ther 2003; 29: 146-56.

3. Davies MD, Daoud MS, Kirby B, Bibson LE, Rogers RS : Clinicopathologic correlation of hypocomplementemic and normocomplementemic urticarial vasculitis. J Am Acad Dermatol 1998; 38: 899-905.

\section{KEY MESSAGES}

- Urticaria and urticarial vasculitis differ in their clinical presentation. The efflorescences of urticarial vasculitis persist for more than 24 hours and leave a brownish residue. The diagnosis is confirmed by histology.

- Hypocomplementemia and urticarial vasculitis are the key to the diagnosis of hypocomplementemic urticarial vasculitis syndrome.

- Besides the skin, the disease process of this systemic vasculitis often involves the eyes, joints, kidneys, and intestinal tract.

- Awareness of this syndrome enables differentiation from other systemic diseases and avoids superfluous diagnostic procedures.

- The most important differential diagnoses of hypocomplementemic urticarial vasculitis syndrome are systemic lupus erythematosus and the mixed cryoglobulinemias.
4. Mehregan DR, Hall MJ, Gibson LE: Urticarial vasculitis: a histopathologic and clinical review of 72 cases. J Am Acad Dermatol 1992; 26: 441-8.

5. Schwartz HR, Mc Duffie FC, Black LF, Schroeter AL, Conn DL: Hypocomplementemic urticarial vasculitis: association with chronic obstructive pulmonary disease. Mayo Clin Proc 1982; 57: 231-8.

6. Jones RR, Bhogal B, Dash A, Schifferli J: Urticaria and vasculitis: A continuum of histological and and immunopathological chances. Br J Dermatol 1983; 108: 695-703.

7. McDuffie FC, Sams WM, Maldonado JE, Andreini PH, Conn DL, Samayoa EA: Hypocomplementemia with cutaneous vasculitis and arthritis. Possible immune complex syndrome. Mayo Clin Proc 1973; 48: 340-8.

8. Palazzo E, Bourgeois P, Meyer O, de Bandt M, Kazatchkine M, Kahn MF: Hypocomplementemic urticarial vasculitis syndrome, Jaccoud's syndrome, valvulopathy: a new syndromic combination. J Rheumatol 1993; 20: 1236-40.

9. Grimbert P, Scholte K, Buisson C, Desvaux D, Baron C, Pastural $M$ : Renal transplantation in a patient with hypocomplementemic urticarial vasculitis syndrome. Am J Kidney Dis 2001; 37: $144-8$

10. Renard M, Wouters C, Proesmans W: Rapidly progressive glomerulonephritis in a boy with hypocomplementemic urticarial vasculitis. Eur J Pediatr 1998; 157: 243-5.

11. Enriquez R, Sirvent AE, Amoros F, Perez M, Matarredona J, Reyes A: Crescentic membranoproliferative glomerulonephritis and hypocomplementemic urticarial vasculitis. J Nephrol 2005; 18 : 318-22.

12. Sanchez NP, Winkelmann RK, Schroeter AL, Dicken CH: The clinical and histopathologic spectrums of urticarial vasculitis. Study of forty cases. J Am Acad Dermatol 1982; 7: 599-605.

13. Wisnieski JJ, Jones SM: IgG autoantibody to collagen-like region of $\mathrm{C} 1 \mathrm{q}$ in hypocomplementemic urticarial vasculitis syndrome, systemic lupus erythematosus, and 6 other musculoskeletal or rheumatic diseases. J Rheumatol 1992; 19: 884-8.

14. Wisnieski JJ : Urticarial vasculitis. Curr Opin Rheumatol 2000; 12: 24-31.

15. Zeiss CR, Burch FX, Marder RJ, Furey NL, Schmid FR, Gewurz H A hypocomplementemic vasculitic urticarial syndrome: report of four new cases and definition of the disease. Am J Med 1980; 68: 687-95.

16. Mehregan DR, Gibson LE: Pathophysiololgy of urticarial vasculitis Arch Dermatol 1998; 134: 88-9.

17. Venzor JE, Lee WL, Huston DP: Urticarial vasculitis. Clin Rev Allergy Immunol 2002; 23: 201-16.

18. Hawkins PN, Lachmann HJ, Agama E, Mc Dermott MF: Spectrum of clinical features in Muckle-Wells syndrome and response to anakinra. Arthritis Rheum 2004; 50: 607-12.

19. St Clair EW, Mc Callum RM: Cogan`s syndrome. Curr Opin Rheumatol 1999: 11: 47-52.

20. Eiling E, Möller M, Kreiselmaier I, Brasch J, Schwarz T: Schnitzler syndrome: treatment failure to rituximab but response to anakinra. J Am Acad Dermatol 2007: 57: 361-4.

21. Mc Neil DJ, Kinsella TD, Craford AM, Fritzler MJ: The AHA syndrome: arthritis, hives and angioedema. Rheumatol Int 1987; 7: 277-9.

22. Baumgärtel MW: Diagnostik, klinisches Spektrum und Verlauf der Kryoglobulinämie. Eine Untersuchung anhand 1640 konsekutiver Patienten einer hämatoimmunologischen Spezialambulanz. 1998, Dissertation der RWTH Aachen.

23. Davies MDP, Brewer JD: Urticarial vasculitis and hypocomplementemic urticarial vasculitis syndrome. Immunol Allergy Clin N Am 2004: 24: 183-213 
24. Worm M, Sterry W, Kolde G: Mycophenolate mofetil is effective for maintenance therapy of hypocomplementemic urticarial vasculitis. Br J Dermatol 2000; 143: 1324.

25. Nishijima C, Hatta N, Inacki M, Sakai H, Tekehara AK: Urticarial vasculitis in systemic lupus erythemathodes: fair response to prednisolone/dapsone and persitent hypocomplementemia. Eur J Dermatol 1999; 9: 54-6.

\section{Corresponding author}

Dr. med. Martin W. Baumgärtel

1. Medizinische Klinik

St. Franziskus Hospital

Hohenzollernring 72

48145 Münster, Germany

Martin.Baumgaertel@sfh-muenster.de

(2) For e-references please refer to:

Www.aerzteblatt-international.de/ref4609 


\section{REVIEW ARTICLE}

\section{Hypocomplementemic Urticarial Vasculitis Syndrome}

An Interdisciplinary Challenge

Wolfgang Grotz, Hideo A. Baba, Jan U. Becker, Martin W. Baumgärtel

\section{E-REFERENCES}

e1. UpToDate 2008: Urticarial vasculitis. www. uptodate.com/2008

e2. Hochberg MC: Updating the ACR revised criteria for the classification of systemic lupus erythematosus. Arthritis Rheum 1997; 40: 1725. 